TITLE:

\title{
Local potential profiling of operating carbon nanotube transistor using frequency- modulation high-frequency electrostatic force microscopy
}

\section{$\operatorname{AUTHOR}(\mathrm{S}):$}

Ito, Masanao; Kobayashi, Kei; Miyato, Yuji;

Matsushige, Kazumi; Yamada, Hirofumi

\section{CITATION:}

Ito, Masanao ... [et al]. Local potential profiling of operating carbon nanotube transistor using frequency-modulation high-frequency electrostatic force microscopy. Applied Physics Letters 2013, 102(1): 013115.

\section{ISSUE DATE:}

2013-01-10

URL:

http://hdl.handle.net/2433/187963

\section{RIGHT:}

(C) 2013 American Institute of Physics. This article may be downloaded for personal use only. Any other use requires prior permission of the author and the American Institute of Physics. 


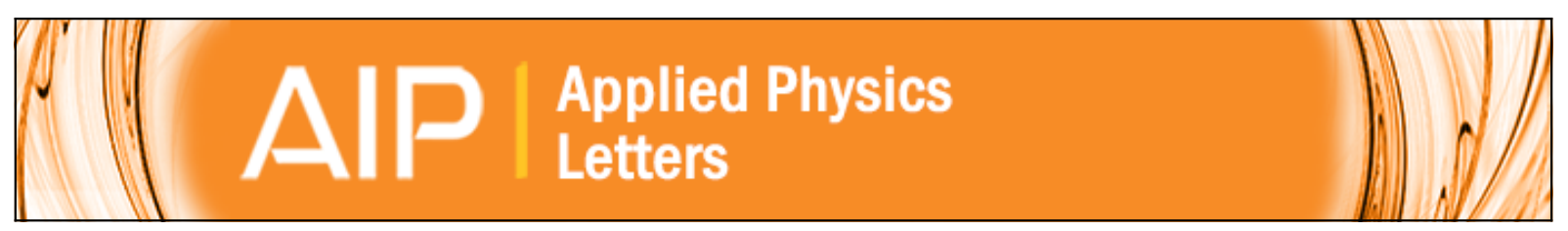

\section{Local potential profiling of operating carbon nanotube transistor using frequency- modulation high-frequency electrostatic force microscopy}

Masanao Ito, Kei Kobayashi, Yuji Miyato, Kazumi Matsushige, and Hirofumi Yamada

Citation: Applied Physics Letters 102, 013115 (2013); doi: 10.1063/1.4774265

View online: http://dx.doi.org/10.1063/1.4774265

View Table of Contents: http://scitation.aip.org/content/aip/journal/apl/102/1?ver=pdfcov

Published by the AIP Publishing

\section{Articles you may be interested in}

High performance transistors via aligned polyfluorene-sorted carbon nanotubes

Appl. Phys. Lett. 104, 083107 (2014); 10.1063/1.4866577

Charge trapping in aligned single-walled carbon nanotube arrays induced by ionizing radiation exposure J. Appl. Phys. 115, 054506 (2014); 10.1063/1.4864126

Extrinsic and intrinsic photoresponse in monodisperse carbon nanotube thin film transistors Appl. Phys. Lett. 102, 083104 (2013); 10.1063/1.4793519

High-frequency performance of scaled carbon nanotube array field-effect transistors

Appl. Phys. Lett. 101, 053123 (2012); 10.1063/1.4742325

Surface potential profiling and contact resistance measurements on operating pentacene thin-film transistors by Kelvin probe force microscopy

Appl. Phys. Lett. 83, 5539 (2003); 10.1063/1.1637443

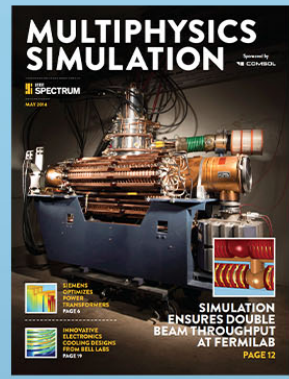

Free online magazine

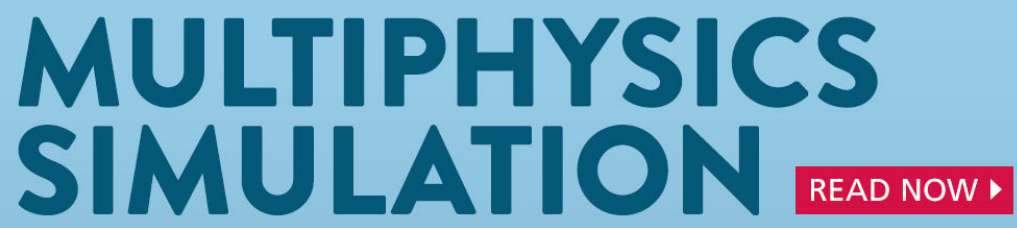




\title{
Local potential profiling of operating carbon nanotube transistor using frequency-modulation high-frequency electrostatic force microscopy
}

\author{
Masanao Ito, ${ }^{1}$ Kei Kobayashi, ${ }^{2, a)}$ Yuji Miyato, ${ }^{1, b)}$ Kazumi Matsushige, ${ }^{1, c)}$ \\ and Hirofumi Yamada ${ }^{1}$ \\ ${ }^{1}$ Department of Electronic Science and Engineering, Kyoto University, Katsura, Nishikyo, \\ Kyoto 615-8510, Japan \\ ${ }^{2}$ Office of Society-Academia Collaboration for Innovation, Kyoto University, Katsura, Nishikyo, \\ Kyoto 615-8520, Japan
}

(Received 14 November 2012; accepted 19 December 2012; published online 10 January 2013)

\begin{abstract}
The local potential measurement of an operating carbon nanotube transistor provides information on the defects in the nanotube and the interfacial potential barriers. While Kelvin-probe force microscopy is a powerful technique to measure the local surface potential, its accuracy is often degraded by the charges trapped on the surrounding insulator surface. Here, we introduce an alternative method to measure the local potential profile along the nanotube being less affected by those charges. We identified the location of a defect and detected the variation in the potential profile for different gate bias voltages, which were not detected by the conventional method. (C) 2013 American Institute of Physics. [http://dx.doi.org/10.1063/1.4774265]
\end{abstract}

Carbon nanotube field-effect transistors (CN-FETs) consisting of a single-walled carbon nanotube (SWNT) ${ }^{1-3}$ have a great potential as future nanoelectronic devices because of their unique electrical properties. Their transport characteristics does not only depend on the intrinsic electronic properties of the SWNT determined by its chirality, but also on the defects in the SWNT and on the potential barriers at the interfaces between the SWNT and electrodes. ${ }^{4-6}$ Local potential measurement of an operating CN-FET provides us information about these limiting factors, therefore, the measurement is of significant importance for improvement of the performance, and thereby for the practical applications of the CN-FETs.

Kelvin-probe force microscopy $(\mathrm{KFM})^{7-9}$ is often utilized to measure the local surface potential of CN-FETs under operating conditions. ${ }^{10-13}$ For the KFM measurement, a modulation voltage of a frequency $f_{\mathrm{m}}$ is applied between the cantilever tip and surface, and the effective contact potential difference (CPD) between them is compensated by applying a control voltage that nullifies the electrostatic force component at $f_{\mathrm{m}}$. The control voltage is recorded as a "surface potential" signal, which represents the local variation in the CPD. However, when the CN-FET is operating, electric charges are often trapped on the silicon oxide surface around the channel because of a high electric field between the nanotube and gate electrode. These trapped charges degrade the accuracy of the potential profile on the nanotube channel measured by KFM because the electrostatic force between the tip and the trapped charges overwhelms that between the tip and the nanotube. ${ }^{10-15}$

In this study, we propose an alternative technique, frequency-modulation high-frequency electrostatic force microscopy (FM-HF-EFM), to measure the potential profiles

\footnotetext{
${ }^{a)}$ Electronic mail: keicoba@iic.kyoto-u.ac.jp.

${ }^{b}$ Present address: Department of Systems Innovation, Osaka University, Toyonaka 560-8531, Japan.

${ }^{c)}$ Present address: Faculty of Science and Technology, Ryukoku University, Seta, Ohtsu 520-2194, Japan.
}

along a nanotube channel as it is less affected by the trapped charges. In this method, a high-frequency modulation voltage is applied only between the tip and nanotube, unlike the conventional KFM technique, in which the modulation voltage is applied between the tip and the whole part of the sample including the gate electrode beneath the nanotube and insulator.

Figure 1 shows a schematic of a CN-FET fabricated on a silicon substrate with an oxide layer. A drain voltage $\left(V_{\mathrm{DS}}\right)$ and gate voltage $\left(V_{\mathrm{GS}}\right)$ are applied to the drain and back gate electrodes, respectively, while the source electrode is electrically grounded, as depicted by the thin solid lines in Fig. 1. The electrostatic force between a cantilever tip, which is electrically grounded, and a sample surface $\left(F_{\mathrm{ES}}\right)$ is generally described as

$$
F_{\mathrm{ES}}=\frac{1}{2} \frac{\partial C_{\mathrm{ts}}}{\partial z}\left(V_{\mathrm{ts}}+V_{\mathrm{CPD}}\right)^{2}
$$

where $V_{\mathrm{ts}}$ and $V_{\mathrm{CPD}}$ are a voltage applied to the sample and the CPD, and $z$ and $C_{\mathrm{ts}}$ are the distance and capacitance between them, respectively. Using the KFM setup, the CPD can be compensated and Eq. (1) reduces to

$$
F_{\mathrm{ES}}=\frac{1}{2} \frac{\partial C_{\mathrm{ts}}}{\partial z} V_{\mathrm{ts}}^{2}
$$

However, in the conventional KFM, the modulation voltage is applied to all electrodes including the back gate electrode, thereby the charges trapped on the insulator strongly affect the $F_{\mathrm{ES}}$ and the measured CPD. The effect of the trapped charges on $F_{\mathrm{ES}}$ can be removed by applying the modulation voltage only to the nanotube channel of concern, which bridges the gap between the source and drain electrodes. We consider the case when an alternating voltage of $V_{\mathrm{ac}} \cos 2 \pi f_{\mathrm{ac}} t$ is applied to the drain electrode, which is equivalent to switching the drain voltage between $+V_{\mathrm{ac}}$ and $-V_{\mathrm{ac}}$ at the frequency $f_{\mathrm{ac}}$. In this case, the effective magnitude of the voltage modulation drops from $V_{\mathrm{ac}}$ at the drain electrode to zero at the source electrode. 


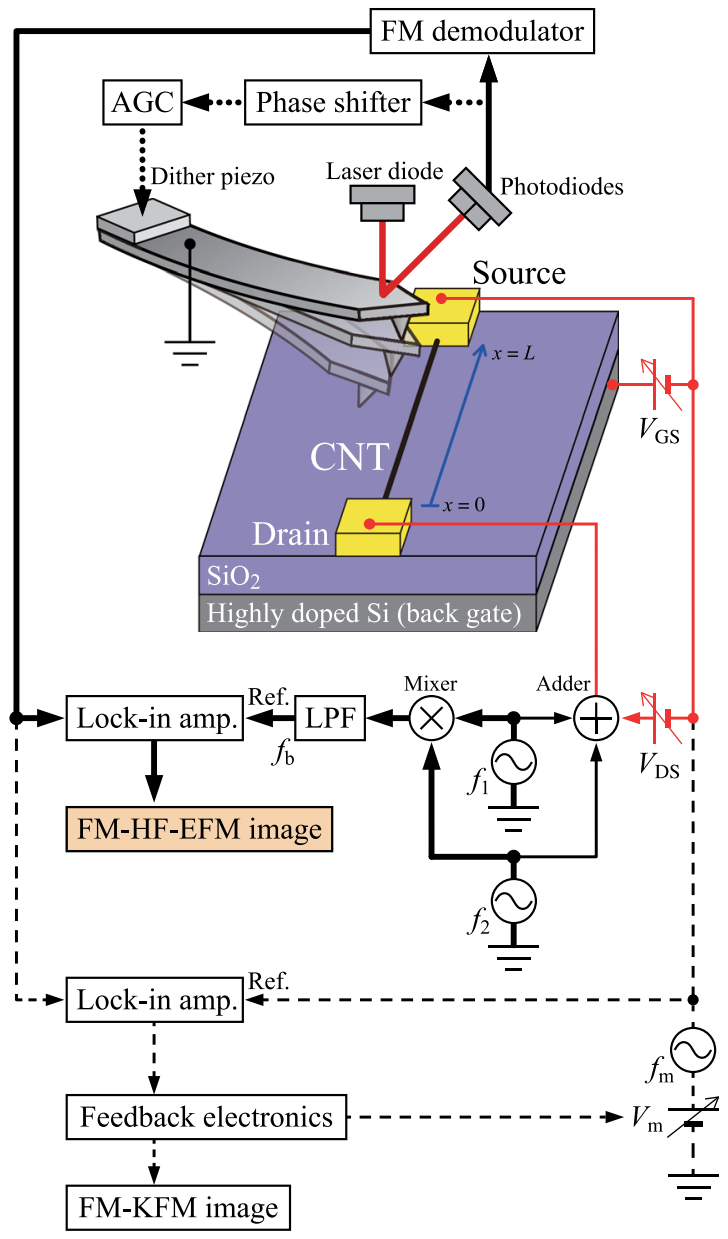

FIG. 1. Schematic diagram of surface potential profiling of CN-FET by FM-HF-EFM. This method is based on FM-AFM in which a cantilever is oscillated by a self-oscillation loop, as indicated by the dotted lines. The oscillation amplitude of the tip is kept constant by an automatic gain controller (AGC). A modulation signal for the FM-HF-EFM is applied to the drain electrode of a CN-FET, as indicated by the thick solid lines. In addition, another modulation signal was applied to cancel the CPD by FM-KFM setup as indicated by the dashed lines.

We define $x$ and $L$ as the position along the nanotube with an origin at the drain electrode and the channel length along the nanotube, respectively, then the effective magnitude of the alternating voltage as a function of $x$ can be described as $V_{\text {local }}(x)$. Since $V_{\text {local }}(x)$ gives the potential profile for the condition when a dc bias voltage of $+V_{\mathrm{ac}}$ is applied to the drain electrode, we can obtain the potential profile on the nanotube channel in the operating CN-FET by determining $V_{\text {local }}(x)$.

Using Eq. (2), the second harmonic component $\left(2 f_{\text {ac }}\right)$ in $F_{\mathrm{ES}}$ as a function of $x$ is described by

$$
F_{\mathrm{ES}}^{2 f_{\mathrm{ac}}}(x)=\frac{1}{4} \frac{\partial C_{\mathrm{ts}}}{\partial z}\left[V_{\text {local }}(x)\right]^{2} \cos 2 \pi\left(2 f_{\mathrm{ac}}\right) t
$$

which shows that we can determine $V_{\text {local }}(x)$ by measuring the distribution of the magnitude of $F_{\mathrm{ES}}{ }^{2 f_{\mathrm{ac}}}(x)$ using the boundary conditions $V_{\text {local }}(0)=V_{\text {ac }}$ and $V_{\text {local }}(L)=0$. Note that it is preferable to utilize the FM detection method to detect the local electrostatic force under a tip but not under a whole cantilever. ${ }^{15}$ Since the measured frequency shift modulation at $2 f_{\mathrm{ac}}, \Delta f_{\mathrm{ES}}^{2 f_{\mathrm{ac}}}(x)$, is proportional to $F_{\mathrm{ES}}^{2 f_{\mathrm{ac}}}(x)$, the potential profile can be determined as

$$
V_{\text {local }}(x)=V_{\text {ac }} \sqrt{\left[\Delta f_{\mathrm{ES}}^{2 f_{\text {ac }}}(x) / \Delta f_{\mathrm{ES}}^{2 f_{\text {ac }}}(0)\right]} .
$$

However, there are some limitations with this method. First, $f_{\mathrm{ac}}$ has to be chosen within the frequency-demodulator bandwidth, which is typically on the order of $\mathrm{kHz}$. The frequency has to be chosen high enough so that the charges on the oxide surface are not redistributed in response to the modulation voltage. If we apply a modulation voltage with two frequency components, $f_{1}$ and $f_{2}$, which are close to each other as depicted by the thick solid lines in Fig. 1, the beat frequency component at $f_{\mathrm{b}}=\left(f_{2}-f_{1}\right)$ is expected in $F_{\mathrm{ES}}{ }^{16}$ When an alternating voltage described as $(1 / 2) V_{\text {ac }}\left(\cos 2 \pi f_{1} t+\cos 2 \pi f_{2} t\right)$ is applied to the drain electrode, the beat component in $F_{\mathrm{ES}}$ becomes

$$
F_{\mathrm{ES}}^{f_{\mathrm{b}}}(x)=\frac{1}{8} \frac{\partial C_{\mathrm{ts}}}{\partial z}\left[V_{\text {local }}(x)\right]^{2} \cos 2 \pi f_{\mathrm{b}} t,
$$

and the potential profile can be calculated in the same way as

$$
V_{\text {local }}(x)=V_{\mathrm{ac}} \sqrt{\left[\Delta f_{\mathrm{ES}}^{f_{\mathrm{b}}}(x) / \Delta f_{\mathrm{ES}}^{f_{\mathrm{b}}}(0)\right]} .
$$

The modulation frequencies can then be arbitrarily chosen as long as $f_{\mathrm{b}}$ is within the bandwidth of the frequency demodulator, and the method also allows us to measure the highfrequency transport characteristics of the CN-FET.

Second, it was assumed that the tip-sample capacitance $\left(C_{\mathrm{ts}}\right)$ is independent of $x$, which is not practical. For example, since the width of the electrodes is usually wider than the width of the SWNT, the effective area of the electrode under the tip is varied when the tip is scanned over the CN-FET. To take the local variation in $C_{\mathrm{ts}}$ into account, Eq. (5) should be rewritten as

$$
F_{\mathrm{ES}}^{f_{\mathrm{b}}}(x)=\frac{1}{8} \frac{\partial}{\partial z}\left\{\int_{S} c_{\mathrm{ts}}(x)\left[V_{\text {local }}(x)\right]^{2} d S\right\} \cos 2 \pi f_{\mathrm{b}} t,
$$

where $c_{\mathrm{ts}}(x)$ and $S$ are the infinitesimal capacitance and effective interaction area beneath the tip. If we consider that the dimensions of the effective interaction area under the tip are smaller than the inverse of the spatial frequency of variation in $V_{\text {local }}(x)$, in the case of the electrostatic force detection using the FM detection technique, Eq. (7) can be simplified as

$$
\begin{aligned}
F_{\mathrm{ES}}^{f_{\mathrm{b}}}(x) & \approx \frac{1}{8} \frac{\partial}{\partial z}\left\{\int_{S} c_{\text {ts }}(x) d S\right\}\left[V_{\text {local }}(x)\right]^{2} \cos 2 \pi f_{\mathrm{b}} t \\
& =\frac{1}{8} \frac{\partial C_{\text {local }}(x)}{\partial z}\left[V_{\text {local }}(x)\right]^{2} \cos 2 \pi f_{\mathrm{b}} t .
\end{aligned}
$$

We can determine the derivative of the local tip-sample capacitance $\left(\partial C_{\text {local }}(x) / \partial z\right)$ by applying the same modulation voltage to both the source and drain electrodes, namely, by setting $V_{\text {local }}(x)=V_{\mathrm{ac}}$ (constant). In this case, $F_{\mathrm{ES}}{ }^{f_{\mathrm{b}}}(x)$ is proportional to the local tip-sample capacitance derivative as

$$
F_{\mathrm{ES}}^{f_{\mathrm{b}}(\text { corr })}(x)=\frac{1}{8} \frac{\partial C_{\text {local }}(x)}{\partial z} V_{\mathrm{ac}}^{2} \cos 2 \pi f_{\mathrm{b}} t,
$$

therefore, $\partial C_{\text {local }}(x) / \partial z$ can be determined by detecting the $f_{\mathrm{b}}$ component. ${ }^{17}$ The corrected potential profile is then obtained by 


$$
V_{\text {local }}^{\text {corr }}(x)=V_{\mathrm{ac}} \sqrt{\left\{\left[\Delta f_{\mathrm{ES}}^{f_{\mathrm{b}}}(x) / \Delta f_{\mathrm{ES}}^{f_{\mathrm{b}}}(0)\right] /\left[\Delta f_{\mathrm{ES}}^{f_{\mathrm{b}}(\text { corr })}(x) / \Delta f_{\mathrm{ES}}^{f_{\mathrm{b}}(\text { corr })}(0)\right]\right\}} .
$$

The third limitation is that one has to apply a modulation voltage with a large voltage swing to the drain electrode to obtain the potential profile corresponding to the operating condition with a large $V_{\mathrm{DS}}$, which reduces the lateral resolution because of the additional long-range electrostatic force. If we assume that $V_{\text {local }}{ }^{\text {corr }}(x)$ linearly changes for a small change in $V_{\text {ac }}$, the potential profile for a large $V_{\mathrm{DS}}$ can also be obtained by adding an offset voltage $V_{\mathrm{dc}}$ to the modulation voltage as

$$
V_{\mathrm{DS}}=V_{\mathrm{dc}}+\frac{1}{2} V_{\mathrm{ac}}\left(\cos 2 \pi f_{1} t+\cos 2 \pi f_{2} t\right) .
$$

For example, the profile for $V_{\mathrm{DS}}=+1 \mathrm{~V}$ can be obtained by applying $V_{\mathrm{dc}}=+0.5 \mathrm{~V}$ and $V_{\mathrm{ac}}=0.5 \mathrm{~V}$, which is reduced by a factor of 2. It should also be noted that the potential for a much larger $V_{\mathrm{DS}}$ can be obtained by summing the profiles collected at an increasing $V_{\mathrm{dc}}$, for example, the profile for $V_{\mathrm{DS}}=+2 \mathrm{~V}$ can be obtained by summing the profiles measured at $V_{\mathrm{dc}}=+0.5 \mathrm{~V}$ and $V_{\mathrm{ac}}=0.5 \mathrm{~V}$ and $V_{\mathrm{dc}}=+1.5 \mathrm{~V}$ and $V_{\mathrm{ac}}=0.5 \mathrm{~V}$.

We now demonstrate the potential profile measurements on a CN-FET using FM-HF-EFM. We fabricated a pair of Pd electrodes on a highly doped silicon substrate with a thermally grown 300-nm-thick silicon dioxide layer. An SWNT isolated by density gradient ultracentrifugation ${ }^{18,19}$ was aligned between the electrodes by dielectrophoresis. ${ }^{20} \mathrm{We}$ used a commercially available AFM instrument (JEOL: JSPM-5200) with home-built electronics. A Pt-coated silicon cantilever (Olympus: OMCL-AC240TM) whose nominal spring constant and resonance frequency were $2 \mathrm{~N} / \mathrm{m}$ and $70 \mathrm{kHz}$, respectively, was used. The cantilever was mechanically oscillated at its resonance frequency and the oscillation amplitude was kept constant at $15 \mathrm{~nm}$ peak-to-peak. A highfrequency signal was generated and the lock-in detection of the beat component was performed by a lock-in amplifier (Zurich Instruments: HF2LI-MF). During the FM-HF-EFM measurement, the CPD was compensated with the FM-KFM setup to suppress any additional long-range electrostatic force which degrades the lateral spatial resolution. All the experiments were performed in a vacuum environment $\left(10^{-4} \mathrm{~Pa}\right)$ at room temperature.

We applied a high-frequency modulation signal of $V_{\mathrm{ac}}=0.5 \mathrm{~V}, f_{1}=5 \mathrm{MHz}$, and $f_{2}=5 \mathrm{M}+500 \mathrm{~Hz}$ with an offset of $V_{\mathrm{dc}}=0.5 \mathrm{~V}$ to the drain electrode. A back gate voltage $V_{\mathrm{GS}}$ was applied to operate the CN-FET. For cancellation of the CPD, another modulation signal of $V_{\mathrm{m}}=0.3 \mathrm{~V}$ and $f_{\mathrm{m}}=1.2 \mathrm{kHz}$ was applied between the tip and the whole part of the sample. We recorded the magnitude of the frequency shift modulation of the cantilever resonance frequency at $f_{\mathrm{b}}$.

Figure 2(a) shows a topographic image of a CN-FET obtained during the FM-HF-EFM measurement, and Figs. 2(b) and 2(c) show FM-HF-EFM images under two different operating conditions; $V_{\mathrm{GS}}$ was set at $-3 \mathrm{~V}$ and $+3 \mathrm{~V}$ for Figs. 2(b) and 2(c), respectively. Since the voltage swing at the drain voltage ranged from $0 \mathrm{~V}$ to $+1 \mathrm{~V}$ by application of the high-frequency signal, the contrasts in the FM-HF-EFM images show the potential profile for $V_{\mathrm{DS}}=+1 \mathrm{~V}$, as mentioned above. The locations of a voltage drop indicated by the arrows were different from each other due to the different gate bias voltages. Figure 2(d) shows an FM-HF-EFM image taken while applying a modulation voltage to both the source and drain electrodes, which represents the variation in the local tip-sample capacitance. We first derived the signal profiles from Figs. 2(b) and 2(c), divided the profiles with the capacitance profile derived from Fig. 2(d), and then the square root of the obtained profiles were obtained. Finally, the potential profile was calibrated using the boundary conditions where the potential at the drain electrode was $+1 \mathrm{~V}$.

Figure 3(a) shows the potential profiles along the SWNT channel calculated from the FM-HF-EFM images. The resultant profile for $V_{\mathrm{GS}}=+3 \mathrm{~V}$ in Fig. 3(a) shows that a large potential drop is observed in the shaded region in the offstate. Figure 3(b) shows the electrical property of the CNFET measured when a dc bias voltage of $+1 \mathrm{~V}$ was applied to the drain electrode, which shows a p-type semiconducting characteristic. The figure shows that the drain current was about $1 \mathrm{nA}$ during the on-state $\left(V_{\mathrm{GS}}=-3 \mathrm{~V}\right)$ and it became less than $10 \mathrm{pA}$ during the off-state $\left(V_{\mathrm{GS}}=+3 \mathrm{~V}\right)$. The total channel resistance in the off-state increased from that in the on-state by a factor of about one hundred. From the results shown in Figs. 3(a) and 3(b), it is considered that a defect exists at the point where the large potential drop was observed and that the defect resistance increased and became dominant in the off-state $\left(V_{\mathrm{GS}}=+3 \mathrm{~V}\right)$.

Finally, Fig. 4 shows "surface potential" images obtained by the conventional FM-KFM. Figure 4(a) is a topographic image of the same sample obtained during the FM-KFM measurement. Figures 4(b) and 4(c) are conventional FM-KFM images acquired under the same bias conditions as the FM-HFEFM measurements; namely, Figs. 4(b) and 4(c) were taken

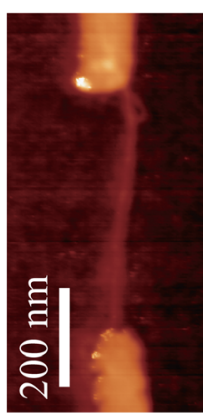

(a)

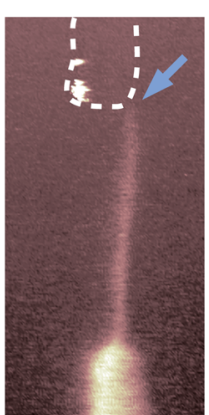

(b)

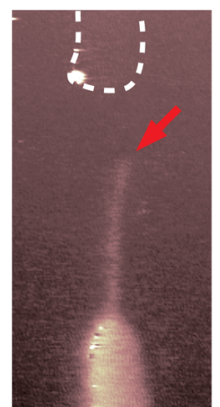

(c)

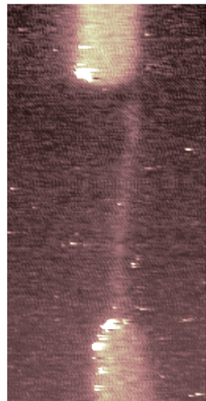

(d)
FIG. 2. (a) Topographic image of CN-FET simultaneously obtained with FM-HF-EFM image obtained while applying $-3 \mathrm{~V}$ to the back gate (onstate) in (b). (c) FM-HF-EFM image obtained while applying $+3 \mathrm{~V}$ to the back gate (off-state). Both (b) and (c) were obtained at $V_{\mathrm{ac}}=0.5 \mathrm{~V}$ with an offset of $V_{\mathrm{dc}}=0.5 \mathrm{~V}$, which correspond to $V_{\mathrm{DS}}=+1 \mathrm{~V}$. (d) Local tip-sample capacitance derivative image for correction of the capacitance variation, which was recorded by applying a modulation voltage for the FM-HF-EFM both to the source and drain electrodes. 


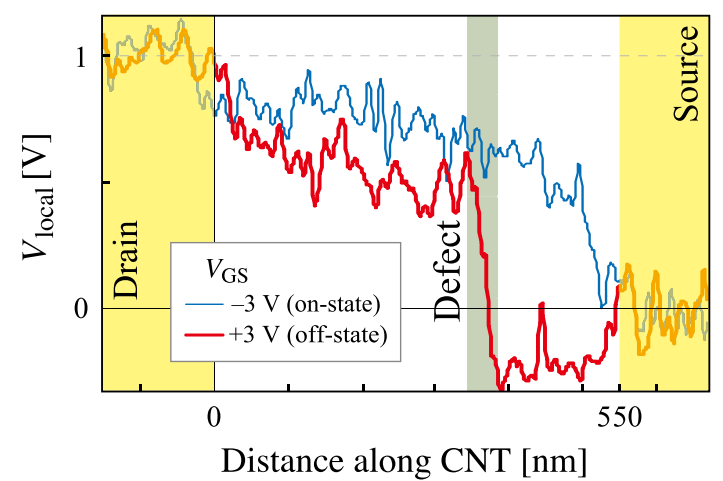

(a)

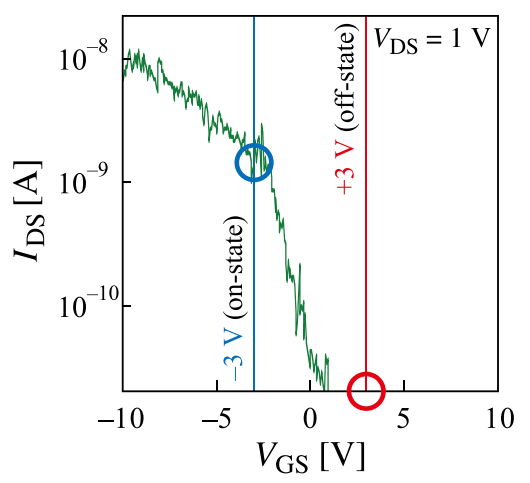

(b) during the on-state $\left(V_{\mathrm{GS}}=-3 \mathrm{~V}\right)$ and off-state $\left(V_{\mathrm{GS}}=+3 \mathrm{~V}\right)$, respectively. The FM-KFM images show local CPD variations with respect to the tip. In Fig. 4(b), a bright contrast was observed because of the positive charges trapped on the oxide surface surrounding the SWNT channel, while a dark contrast was observed for the area away from the channel or the electrodes because of the negative gate bias voltage. In Fig. 4(c), the image contrasts on the oxide surface were almost inverted, and the locations of the potential drop are not clear in either conventional FM-KFM images unlike FM-HF-EFM images shown in Figs. 2(b) and 2(c). Figure 4(d) shows the potential profiles along the SWNT channel taken from Figs. 4(b) and 4(c). The distinct difference of potential distribution between the two states, which was observed in Fig. 3(a), cannot be recognized in Fig. 4(d), owing to the charges trapped on the oxide around the SWNT channel.

The FM-HF-EFM showed the defect in the channel, while the FM-KFM images showed the trapped charges on the silicon oxide. We assume that the on/off characteristic of the CN-FET presented here was governed by the defect in the CNT, while the trapped charges caused the hysteresis nature of the gate voltage dependence (not shown). Both techniques give us complementary information to understand the transport characteristics of the CN-FET.

Note that the profiles obtained by FM-HF-EFM were noisier than those by FM-KFM, and they show even negative potentials in off-state, which are caused by the division of the measured profiles by the local capacitance profile measured with a different scan. In the near future, these profiles could be acquired in a single scan by changing the bias setting at each pixel using an advanced control software, which would improve the signal-to-noise ratio in the calculated potential profiles.

In conclusion, we developed a method for surface potential investigations of CN-FET devices, FM-HF-EFM, which is an alternative to the conventional FM-KFM technique. We visualized the location of a defect in an operating CN-FET using the FM-HF-EFM. The potential profiles along the channel revealed the dependence of the location of a potential drop on the gate voltage, which was not detected by the

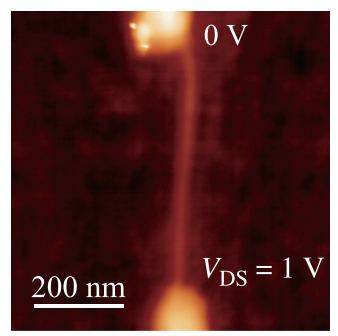

(a)

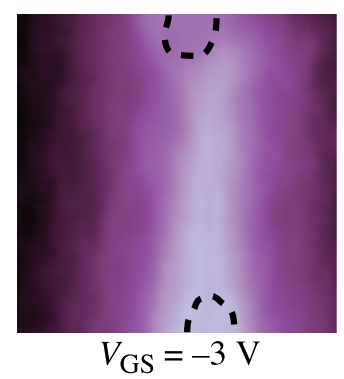

(b)

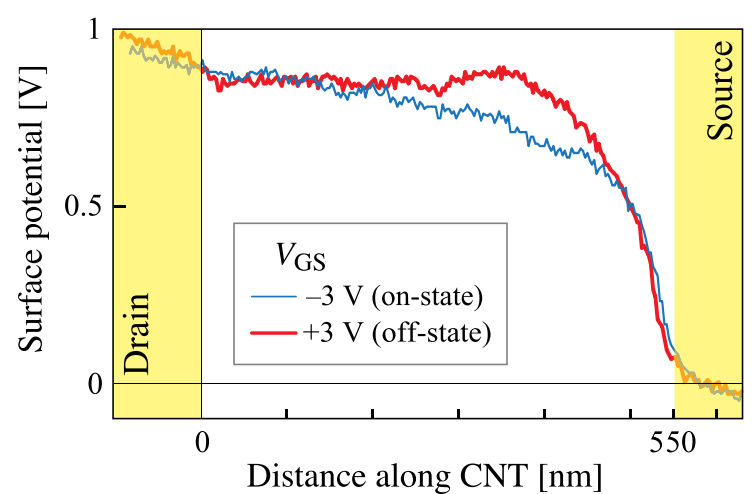

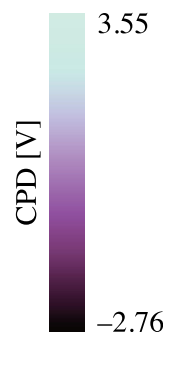

(c)

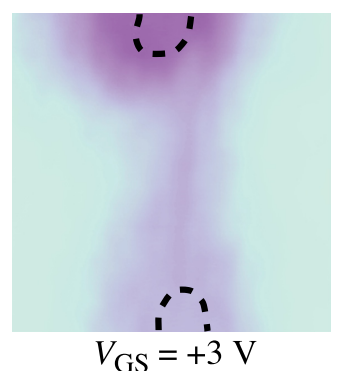

FIG. 4. (a) Topographic image of the same CNFET. (b) Conventional FM-KFM image obtained while applying $-3 \mathrm{~V}$ to the back gate (on-state). (c) Conventional FM-KFM image obtained while applying $+3 \mathrm{~V}$ to the back gate (off-state). (d) Potential profiles along the SWNT channel taken from (b) and (c).

(d) 
conventional FM-KFM technique. The results demonstrated that the accuracy of the potential profile was improved by the FM-HF-EFM compared to the conventional FM-KFM because of the potential profiling with the FM-HF-EFM is less affected by the charges trapped on the insulating oxide surface surrounding the nanotube channel. The technique presented here, although its signal-to-noise ratio can be improved, is a promising technique to investigate local electronic properties of one-dimensional semiconductors, such as silicon nanowires and molecular wires, whose widths are on the order of nanometers, with the capability of highfrequency measurements.

This study was supported in part by a Grant-in-Aid for Scientific Research on Priority Areas (Grant No. 19054010) and by the Global Center of Excellence (G-COE) Program of the Ministry of Education, Culture, Sports, Science and Technology of Japan (MEXT).

${ }^{1}$ S. J. Tans, A. R. M. Verschueren, and C. Dekker, Nature 393, 49 (1998). ${ }^{2}$ R. Martel, T. Schmidt, H. R. Shea, T. Hertel, and Ph. Avouris, Appl. Phys. Lett. 73, 2447 (1998).

${ }^{3}$ M. Burghard, H. Klauk, and K. Kern, Adv. Mater. 21, 2586 (2009), and references therein.

${ }^{4}$ S. Heinze, J. Tersoff, R. Martel, V. Derycke, J. Appenzeller, and Ph. Avouris, Phys. Rev. Lett. 89, 106801 (2002).
${ }^{5}$ J. Appenzeller, J. Knoch, V. Derycke, R. Martel, S. Wind, and Ph. Avouris, Phys. Rev. Lett. 89, 126801 (2002).

${ }^{6}$ M. Freitag, A. T. Johnson, S. V. Kalinin, and D. A. Bonnell, Phys. Rev. Lett. 89, 216801 (2002).

${ }^{7}$ J. M. R. Weaver and D. W. Abraham, J. Vac. Sci. Technol. B 9, 1559 (1991).

${ }^{8}$ M. Nonnenmacher, M. P. O'Boyle, and H. K. Wickramasinghe, Appl. Phys. Lett. 58, 2921 (1991).

${ }^{9}$ S. Kitamura and M. Iwatsuki, Appl. Phys. Lett. 72, 3154 (1998).

${ }^{10}$ A. Bachtold, M. S. Fuhrer, S. Plyasunov, M. Forero, E. H. Anderson, A. Zettl, and P. L. McEuen, Phys. Rev. Lett. 84, 6082 (2000).

${ }^{11}$ Y. Miyato, K. Kobayashi, K. Matsushige, and H. Yamada, Jpn. J. Appl. Phys., Part 1 44, 1633 (2005).

${ }^{12}$ T. Umesaka, H. Ohnaka, Y. Ohno, S. Kishimoto, K. Maezawa, and T. Mizutani, Jpn. J. Appl. Phys., Part 1 46, 2496 (2007).

${ }^{13}$ D. Brunel, D. Deresmes, and T. Mélin, Appl. Phys. Lett. 94, 223508 (2009).

${ }^{14}$ H. G. Ong, J. W. Cheah, L. Chen, H. TangTang, Y. Xu, B. Li, H. Zhang, L.-J. Li, and J. Wang, Appl. Phys. Lett. 93, 093509 (2008).

${ }^{15}$ M. Ito, Y. Hosokawa, R. Nishi, Y. Miyato, K. Kobayashi, K. Matsushige, and H. Yamada, e-J. Surf. Sci. Nanotechnol. 9, 210 (2011), and references therein.

${ }^{16}$ A. S. Hou, F. Ho, and D. M. Bloom, Electron. Lett. 28, 2302 (1992).

${ }^{17}$ Y. Martin, D. W. Abraham, and H. K. Wickramasinghe, Appl. Phys. Lett. 52, 1103 (1988).

${ }^{18}$ M. S. Arnold, A. A. Green, J. F. Hulvat, S. I. Stupp, and M. C. Hersam, Nature Nanotech. 1, 60 (2006).

${ }^{19}$ K. Kaneko, Y. Miyato, K. Kobayashi, K. Matsushige, and H. Yamada, Jpn. J. Appl. Phys., Part 1 49, 02BD04 (2010).

${ }^{20}$ X. Q. Chen, T. Saito, H. Yamada, and K. Matsushige, Appl. Phys. Lett. 78, 3714 (2001) 